\title{
Saúde do trabalhador nos estudos de impactos de refinarias de petróleo
} Health worker in studies of impacts oil refinery

\author{
José Marcos da Silva ${ }^{1}$, Lia Giraldo da Silva Augusto², Idê Dantas Gurgel ${ }^{3}$
}

\begin{abstract}
Resumo
Este artigo apresenta a análise da saúde do trabalhador no licenciamento de refinarias de petróleo no Brasil. Utilizou-se a análise documental partindo de um marco referencial que inter-relaciona saúde do trabalhador e refino de petróleo, levantando-se 30 conteúdos de saúde do trabalhador a serem verificados nos Estudo de Impactos Ambientais (EIA). Desenvolveu-se o indicador de grau de inserção (GI), segundo as dimensões: incipiente, insuficiente, intermediário ou avançado. Identificou-se o grau de inserção incipiente (GI incipiente - 0 a 20\%) para o EIA REFINE e o EIA COMPERJ, e insuficiente para o EIA REPLAN (GI insuficiente - 25 a 45\%). Em relação ao grau de inserção total, o EIA REPLAN apresentou o grau insuficiente (30\%), seguido do EIA COMPERJ e do EIA REFINE, que apresentaram o grau incipiente (13\%; $23 \%$ ). Ante os resultados encontrados, sugere-se fortemente a inserção de conteúdos de saúde do trabalhador nos ElAs de refinarias como estratégia de vigilância em saúde e controle de riscos.
\end{abstract}

Palavras-chave: saúde do trabalhador; vigilância em saúde do trabalhador; trabalhadores.

\section{Abstract}

This article presents the analysis of occupational health in the licensing of oil refineries in Brazil. The documentary analysis was applied starting from a reference point that interrelates worker health and oil refining, rising 30 contents employee health to be checked in EIA. An indicator of the degree of integration (DI) was developed, according to the dimensions: incipient, insufficient, intermediate or advanced. The degree of incipient insertion (DI incipient - 0 to 20\%) was identified for the EIA REFINE and COMPERJ and insufficient for EIA REPLAN (DI insufficient - 25 to $45 \%$ ). Regarding to the degree of full insertion, the EIA REPLAN presented insufficient degree (30\%), followed by the EIA COMPERJ and EIA REFINE which presented the incipient degree $(13 \%, 23 \%)$. Face these results, the insertion of worker health contents in EIA refineries is strongly recommended as a strategy for health surveillance and risk control.

Keywords: occupational health; surveillance of the workers health; workers.

Trabalho realizado no Laboratório de Saúde, Ambiente e Trabalho - LASAT/CPqAM/FIOCRUZ - Recife (PE), Brasil.

${ }^{1}$ Mestre em Ciências; Pesquisador do Laboratório Saúde Ambiente e Trabalho - Centro de Pesquisas Aggeu Magalhães, FIOCRUZ - Recife (PE), Brasil.

2Doutora em Medicina; Pesquisadora Titular do Centro de Pesquisas Aggeu - Recife (PE), Brasil.

${ }^{3}$ Doutora em Ciências. Pesquisadora Titular do CPqAM, FIOCRUZ - Recife (PE), Brasil.

Endereço para correspondência: José Marcos da Silva - Rua Almirante Saldanha da Gama, 45, apto. 108 - CEP: 51130-220 - Recife (PE), Brasil -

E-mail: kinhoventuras@hotmail.com

Fonte de financiamento: nenhuma.

Conflitos de interesses: nada a declarar. 


\section{INTRODUÇÃO}

A vigilância da saúde do trabalhador tem na vigilância de trabalhadores expostos a solventes aromáticos seu marco inicial, dado pela Secretaria de Saúde do Estado de São Paulo no ano de $1985^{1}$.

Nesse sentido, a Vigilância em Saúde inseriu no seu escopo de atuação ações voltadas a situações que envolvem trabalhadores expostos a produtos químicos nos territórios em que são implantados processos produtivos poluidores.

Certamente, a Rede Nacional de Atenção Integral à Saúde do Trabalhador tem fortalecido os municípios para mudanças nas práticas das organizações de saúde, com o objetivo de qualificar a vigilância em saúde do trabalhador ${ }^{2}$.

A Constituição Federal de 1988 (CF 1988), além de criar o Sistema Único de Saúde (SUS), estabelece a saúde como objetivo final nas situações que envolvem a proteção do ambiente ante os impactos resultantes da implantação de empreendimentos no território ${ }^{3}$.

O Estudo de Impactos Ambientais (EIA) fundamenta o licenciamento de empreendimentos cujo processo produtivo apresente riscos à saúde, tendo como objetivo permitir a viabilidade, ou não, da implantação, contextualizando os impactos às dimensões biológica, física, social e cultural, como exigência da lei 6.938/1981 das resoluções do Conselho Nacional de Meio Ambiente $001 / 1986$ e $237 / 1997^{3,4}$.

De maneira afirmativa, autoridades sanitárias colocam que a saúde deve ser considerada no licenciamento servindo à solução de problemas gerados pela implantação de processos produtivos nos territórios ${ }^{5,6}$.

Nesse sentido, o petróleo ocupa destaque nas discussões públicas pela expectativa do governo brasileiro em manter a autossuficiência na produção, condição alcançada em $2006^{7}$.

Por isso, há a perspectiva de implantação de novas refinarias, que representam potenciais de impactos sobre a saúde dos trabalhadores, por causa das nocividades no ambiente de trabalho pela exposição a hidrocarbonetos aromáticos e a metais pesados ${ }^{8,9}$.

A análise da inserção da saúde do trabalhador nos EIAs de refinarias se torna estratégica porque a indústria petrolífera tem sido reconhecida por seu potencial devastador, afetando diretamente a qualidade do ar, da água, do solo e dos seres vivos - em particular, dos seres humanos ${ }^{10-12}$.

Entre os problemas de saúde relacionados estão: aumento de câncer; incremento das doenças neurológicas e psíquicas; doenças de pele; doenças hepáticas; doenças cardiovasculares; doenças respiratórias que alcançam trabalhadores e comunidades ${ }^{13,14}$.
Estudos consideram o trabalho na indústria do petróleo como sendo uma atividade complexa, contínua, coletiva e perigosa, envolvendo riscos aos trabalhadores ${ }^{9,15}$.

O ambiente de trabalho nas refinarias está marcado por acidentes nas plantas de refinarias e, em sua maioria, são acidentes de engenharia e de manutenção, acidentes típicos de trabalho e acidentes químicos ampliados gerados por explosões, vazamentos, disposição inadequada de resíduos e transporte de produtos perigosos ${ }^{9,12}$.

A forma de enfrentamento para essas realidades precisa ser de atividades variadas, pautadas na vigilância em saúde, para proteção, promoção da saúde dos trabalhadores expostos a riscos e agravos relativos às condições de trabalho ${ }^{3}$.

Partindo do pressuposto de que a inserção da saúde do trabalhador nos EIAs pode ser uma estratégia de prevenção por ser fonte de subsídios para antecipar ações preventivas para os sistemas municipais nos territórios, este estudo analisa a inserção da saúde do trabalhador nos EIAs das refinarias Abreu e Lima (Ipojuca/PE), Paulínia (Campinas/SP) e Complexo Petroquímico do Rio de Janeiro (Itaboraí/RJ).

\section{METODOLOGIA}

Pesquisa avaliativa do tipo análise de inserção ${ }^{16} \mathrm{com}$ vistas a determinar a medida descritiva da inserção da saúde do trabalhador nos EIAs de refinarias, tendo como estratégia o estudo de casos, adotando os princípios da análise documental para interpretação dos dados.

Analisaram-se os EIAs das Refinarias: Paulínia (EIA de aperfeiçoamento de planta); Abreu e Lima (EIA de implantação); e Complexo Petroquímico do Rio de Janeiro (EIA de implantação).

Os critérios da inserção foram levantados em portarias, relatórios, minutas do Ministério da Saúde, teses, dissertações e artigos científicos relacionados à saúde do trabalhador, identificando e caracterizando os conteúdos que aparecem nas tabelas dos resultados. Estabeleceram-se três categorias para análise: diagnóstico, análise e proteção da saúde do trabalhador.

Para a estimação do grau de inserção, atribuíram-se aos critérios a pontuação: 1 (um) para situação favorável à inserção; 0,5 (meio) para situação de inserção parcial; 0 (zero) ausência de conteúdos.

Em seguida, foi estimado o grau de inserção categoria de análise; neste caso o grau de inserção resultou na relação percentual entre a soma das pontuações obtidas e a soma da pontuação máxima, considerados quatro graus para a inserção: avançado 75-100\%; intermediário: 50-75\%; insuficiente: 25-50\%; e incipiente: $0-5 \%$. 
Realizou-se a análise total de inserção (Grau de Inserção Total) por meio da soma da pontuação encontrada para as categorias de análise em cada EIA e o total de pontos alcançáveis. Estabeleceu-se o consenso entre pesquisadores, sob a supervisão de um supervisor sênior para assegurar a vigilância epistemológica. O projeto foi aprovado pelo $\mathrm{CEP} / \mathrm{CPqAM} /$ Fiocruz protocolo no 112/08.

\section{RESULTADOS}

Na Tabela 1, verifica-se que o grau de inserção de conteúdos de diagnóstico foi incipiente em todos os EIAs. Destacase o EIA REPLAN (25\%), por apresentar a maior pontuação no aspecto inventário de substâncias químicas.

Com relação à equipe multiprofissional, os EIAs REPLAN e REFINE apresentaram profissionais com formação inicial na área de saúde. No entanto, os profissionais dessa área não participaram da identificação dos impactos socioeconômicos que incluem os impactos à saúde, o que seria fundamental. O EIA COMPERJ apresentou o menor grau de inserção (10\%).
Quanto ao grau de inserção de aspectos de análise de saúde do trabalhador, observa-se que o EIA REPLAN apresentou grau insuficiente (35\%) e os EIAs COMPERJ e REFINE, grau incipiente (20\%), conforme Tabela 2.

Na Tabela 3 está a distribuição dos resultados de conteúdos de proteção da saúde do trabalhador.

O EIA REPLAN apresentou o grau intermediário (55\%) por inserir itens de segurança no design industrial; no entanto, não apresentou planos de emergência para acidentes ampliados.

O EIA Refine apresentou grau insuficiente (45\%) e o EIA COMPERJ, grau incipiente (10\%). Nenhum documento considerou a utilização de biomarcadores sensíveis para a prevenção de morbidades associadas ao processo de refino do petróleo.

Em relação ao grau de inserção total de saúde do trabalhador (Tabela 4), verifica-se que nenhum dos EIAs estudados inseriram pelo menos $50 \%$ de conteúdos relacionados a Saúde do Trabalhador. Destaca-se o EIA da REPLAN, que, apesar de apresentar grau insuficiente (30\%), superou os demais, principalmente pela introdução de inovações tecnológicas.

Tabela 1. Grau de inserção de conteúdos de diagnóstico de saúde do trabalhador nos ElAs de refinarias, Brasil, 2011

Estudo de impacto

GI incipiente - 10\%

EIA REPLAN

GI incipiente - $20 \%$

EIA REFINE

GI incipiente - $15 \%$

\section{Pontuação}

0 ponto: equipe multiprofissional 0 ponto:identificação de Centro de Referência

0 pontos: lista de agravos de notificação 0 ponto: perfil de morbimortalidade

0 ponto: envolvimento do controle social 0 ponto: demandas de atenção à saúde do trabalhador

0,5 ponto: inventário de substâncias químicas

0 ponto: contextualização de situações de riscos

0 ponto: protocolos de doenças relacionadas ao trabalho

0,5 ponto: identificação da cobertura assistencial SUS

0,5 ponto: equipe multiprofissional

0 ponto: identificação de Centro de Referência

0 pontos: lista de agravos de notificação

0 ponto: perfil de morbimortalidade

0 ponto: envolvimento do controle social

0 ponto: demandas de atenção à saúde do trabalhador

0,5 ponto: inventário de substancias químicas

0,5 ponto: contextualização de situações de riscos

0 ponto: protocolos de doenças relacionadas ao trabalho

0 ponto: identificação da cobertura assistencial SUS

0,5 ponto: equipe multiprofissional

0 ponto: identificação de Centro de Referência

0 pontos: lista de agravos de notificação

0 ponto: perfil de morbimortalidade

0 ponto: envolvimento do controle social 0 ponto: demandas da atenção à Saúde do trabalhador

0,5 ponto: inventário de substâncias químicas

0 ponto: contextualização de situações de riscos

0 ponto: protocolos de doenças relacionadas ao trabalho

0,5 ponto: identificação da cobertura assistencial SUS

Gl: grau de inserção. 
Tabela 2. Grau de inserção de aspectos relacionados à análise de saúde do trabalhador nos ElAs de refinarias, Brasil, 2011 Estudo de impacto Grau de inserção

Pontuação

0 ponto: participação social

0 ponto: percepção social dos riscos e benefícios

1 ponto: estimativa positiva - empregos

0 ponto: estimativa negativa - novos agravos

0,5 ponto: exposição a poluentes químicos

EIA COMPER

GI incipiente - $20 \%$

0,5 ponto: uso de indicadores da saúde pública

0 ponto: identificação de grupos de trabalhadores vulneráveis

0 ponto: desenvolvimento de cenários de riscos de acidentes de trabalho

0 ponto: identificação de programas de saúde do trabalhador SUS

0 ponto: existência de estratégias compartilhadas (público-privado)

0 ponto: participação social

0,5 ponto: percepção social dos riscos e benefícios

1 ponto: estimativa positiva - empregos

0 ponto: estimativa negativa - novos agravos

EIA REPLAN $\quad$ GI insuficiente - 35\%

0,5 ponto: presunção de exposição a poluentes químicos

0,5 ponto: uso de indicadores da saúde pública

0 ponto: identificação de grupos de trabalhadores vulneráveis

0 ponto: desenvolvimento de cenários de riscos de acidentes de trabalho

0 ponto: identificação de programas de saúde do trabalhador SUS

1 ponto: existência de estratégias compartilhadas (público-privado)

0 ponto: participação social

0 ponto: percepção social dos riscos e benefícios

1 ponto: estimativa positiva - empregos

0 ponto: estimativa negativa - novos agravos

0,5 ponto: exposição a poluentes químicos

0,5 ponto: uso de indicadores da saúde pública

EIA REFINE GI incipiente - $20 \%$

0 ponto: identificação de grupos de trabalhadores vulneráveis 0 ponto: desenvolvimento de cenários de riscos de acidentes de trabalho

0 ponto: identificação de programas de saúde do trabalhador SUS

0 ponto: existência de estratégias compartilhadas (público-privado)

Gl: grau de inserção.

\section{DISCUSSÃO}

Em relação ao diagnóstico da saúde do trabalhador, um aspecto importante deve ser a participação de profissional com qualificação e formação específica na área de saúde, integrando equipes responsáveis pela elaboração dos EIAs. Nesse sentido, constatou-se que nos EIAs REPLAN e COMPERJ as questões e os impactos à saúde não foram identificados por profissionais com qualificação técnica específica, o que pode ter contribuído para a incipiência das informações.

Do mesmo modo, essa ausência pode refletir o distanciamento entre o licenciamento ambiental e o setor da saúde, seja pela ausência de profissionais de saúde atuando nas equipes dos órgãos ambientais que orientaram a elaboração dos respectivos EIAs ou, até mesmo, por falhas na formação dos profissionais que atuam nas empresas de consultoria.

O acúmulo de conhecimento desenvolvido no SUS sobre a saúde do trabalhador pode contribuir tanto para a formação de recursos humanos para o olhar da saúde no processo de licenciamento como para o esclarecimento à população sobre os impactos, fortalecendo o debate qualificado do controle social nas audiências públicas previstas por ocasião da implantação de refinarias.
Mesmo no EIA REFINE, do qual participou um farmacêutico, as informações sobre saúde do trabalhador são quase inexistentes. Autores apontam que o predomínio de consultores da biologia e da engenharia parece concentrar as informações em dois pólos: um pólo biológico (foco na flora e na fauna); e outro pólo das coisas inanimadas ou plantas de engenharia, carregado de informações sobre as unidades de produção, projetos de manutenção e plantas industriais ${ }^{17,18}$.

Segundo Porto e Milanez ${ }^{17}$, torna-se imprescindível a participação de ecologistas, sanitaristas, sociólogos, geógrafos, antropólogos, que poderiam analisar os eventos na perspectiva da coletividade, com base no diagnóstico da situação de saúde, utilizando abordagens integradas e superando os limites da engenharia e da biologia conservacionista.

A situação de saúde deveria orientar a proposição de medidas de proteção à saúde dos trabalhadores. Isso é possível, tomando por base informações oriundas de produções acadêmicas, além do uso de bancos de dados, antecipando mudanças.

O grau de inserção sobre o diagnóstico de saúde do trabalhador demonstra a falta de preocupação com grupos vulnerabilizados e com acidentes característicos das refinarias ${ }^{9}$. 
Tabela 3. Grau de inserção de aspectos relacionados à proteção da saúde do trabalhador nos EIAs de refinarias, Brasil, 2011 Estudo de impacto $\quad$ Grau de inserção $\quad$ Pontuação

0 ponto: uso de biomarcadores sensíveis - moleculares, celulares

0,5 ponto: tecnologias de proteção e segurança coletiva 0,5 ponto: medidas de segurança individual 0 ponto: inovação no design industrial

EIA COMPERJ GI incipiente - $10 \%$

0 ponto: tecnologias de gerenciamento de riscos

\begin{tabular}{|c|c|c|}
\hline & & $\begin{array}{c}0 \text { ponto: sistema de acompanhamento } \\
0 \text { ponto: método de acompanhamento } \\
0 \text { ponto: capacidade institucional } \\
0 \text { ponto: serviço de referência especializada de atenção à saúde } \\
0 \text { ponto: indicadores de acompanhamento das situações de riscos }\end{array}$ \\
\hline EIA REPLAN & GI insuficiente - 35\% & $\begin{array}{c}0 \text { ponto: uso de biomarcadores sensíveis - moleculares, celulares } \\
0,5 \text { ponto: tecnologias de proteção e segurança coletiva } \\
1 \text { ponto: medidas de segurança individual } \\
1 \text { ponto: inovação no design industrial } \\
1 \text { ponto: tecnologias de gerenciamento de riscos } \\
0 \text { ponto: sistema de acompanhamento } \\
0 \text { ponto: método de acompanhamento } \\
0 \text { ponto: capacidade institucional } \\
0 \text { ponto: apresentação dos planos de emergência e acidentes ampliados } \\
0 \text { ponto: serviços de referência especializada de atenção à saúde } \\
0 \text { ponto: indicadores de acompanhamento das situaçoses de riscos }\end{array}$ \\
\hline EIA REFINE & GI insuficiente - $35 \%$ & $\begin{array}{c}0 \text { ponto: uso de biomarcadores sensíveis - moleculares, celulares } \\
0,5 \text { ponto: tecnologias de proteção e segurança coletiva } \\
1 \text { ponto: medidas de segurança individual } \\
1 \text { ponto: inovação no design industrial } \\
1 \text { ponto: tecnologias de gerenciamento de riscos } \\
0 \text { ponto: planos de emergência e acidentes ampliados } \\
0 \text { ponto: sistema de acompanhamento } \\
0 \text { ponto: método de acompanhamento } \\
0 \text { ponto: capacidade institucional } \\
0 \text { ponto: serviços de referência especializada } \\
0 \text { ponto: indicadores de acompanhamento }\end{array}$ \\
\hline
\end{tabular}

Gl: grau de inserção.

Tabela 4. Grau de inserção total de saúde do trabalhador nos EIAs de refinarias, Brasil, 2011

\begin{tabular}{|c|c|c|c|c|}
\hline \multirow[t]{2}{*}{ Estudo de impacto } & \multicolumn{3}{|c|}{$\begin{array}{l}\text { Conteúdo sobre saúde do trabalhador } \\
\text { Diagnóstico análise proteção }\end{array}$} & \multirow[t]{2}{*}{ Grau total } \\
\hline & Diagnóstico & Análise & Proteção & \\
\hline COMPERJ & 1 & 2 & 1 & GIT - incipiente - $13 \%$ \\
\hline REPLAN & 2 & 3,5 & 3,5 & GIT - insuficiente - 30\% \\
\hline REFINE & 1,5 & 2 & 3,5 & GIT - incipiente - 23\% \\
\hline
\end{tabular}

GIT: grau de inserção total.

Para Augusto $^{19}$, essas informações são essenciais em contextos de desenvolvimento industrial porque ocorre a migração, que responde ao aumento da demanda por serviços de saúde, já que aumenta o número de famílias nos territórios, o que impõe a necessidade de ampliação da cobertura dos programas saúde da família, entre outras pressões.

Em relação à análise de saúde do trabalhador, a participação social na análise de situações de vulnerabilidade aponta para o conceito de sustentabilidade socioambiental. No entanto, os EIAs analisados demonstram a falta de compromisso social ao desconsiderarem o fato de que o conhecimento daqueles que estarão expostos contribui para uma análise próxima da realidade.

Nesse contexto, envolver é o modo eficiente para a análise das questões de saúde, visando à identificação de contextos de riscos e de ameaças ${ }^{19}$.

Apesar de utilizarem indicadores da saúde pública, estes são descontextualizados dos riscos introduzidos pelo processo de refino do petróleo. Segundo Mariano ${ }^{11}$, o processo de refino contamina os compartimentos ambientais (água, ar e solo) e a cadeia alimentar, alcançando os seres humanos. 
Uma atividade imprescindível em relação aos impactos à saúde pela exposição a substâncias químicas tem sido o inventário de substâncias químicas. Os EIAs apontaram as substâncias envolvidas no refino, mas não estabelecem relação entre a substância e os riscos à saúde do trabalhador ${ }^{11,12}$.

Nenhuma consideração foi ser feita aos danos e agravos aos trabalhadores, apesar da literatura inferir situações de vulnerabilização e mudanças no perfil de morbimortalidade ${ }^{8,9,13,14}$.

Augusto $^{13,14}$ mostrou associação entre exposição de trabalhadores a poluentes químicos presentes na cadeia produtiva do petróleo com processos fisiopatológicos responsáveis por doenças hematológicas e alterações genotóxicas.

Em relação ao impacto causado a outras cadeias produtivas e aos modos de trabalho existentes no território, no EIA REFINE há a referência à falta de compreensão dos trabalhadores (pescadores) da praia de SUAPE, na cidade do Cabo de Santo Agostinho. Segundo os consultores que elaboraram o EIA, esses trabalhadores se consideram donos do local, sugerindo como solução um esclarecimento social quanto à maneira como os pescadores podem mudar sua atividade, já que o tráfego de navios petroleiros, que vão escoar os derivados da refinaria, comprometerá a pesca tradicional. De forma indireta, sugerem que os pescadores comprem barcos potentes o suficiente para pescar em alto mar, longe do porto de Suape.

Diversos autores apontam esse discurso como resultado de um modelo de desenvolvimento que exclui e inviabiliza grupos humanos, pela desconsideração da realidade histórica das pessoas com o seu território ${ }^{20,21}$.

Pode-se considerar, com base em Augusto ${ }^{1}$, que conflitos socioambientais estão inter-relacionados e indissociáveis das questões de saúde e que diversos conflitos relacionados à saúde do trabalhador resultam de realidades marcadas por dominação política e apropriação econômica.

Porto ${ }^{20}$ revela o uso de discursos favoráveis ao crescimento econômico, em que se formam consensos artificiais que impõem a ideia de fatalidade de situações de violação de direitos como sendo um mal necessário para o bem da maioria.

Isso contribui para que os acidentes de trabalho sejam desconsiderados, da mesma forma que inexistem informações sobre os grupos de trabalhadores mais vulnerabilizados, mesmo sendo conhecidos pelos riscos de explosões nas atividades operacionais pertinentes ao trabalho dos petroleiros?

Todos os EIAs realizaram levantamento da estimativa de impactos positivos. Entretanto, o mesmo não foi realizado com relação aos danos à saúde do trabalhador. Nenhum dos
EIAs apresenta informações contextualizadas sobre isso e sobre os cenários prospectivos de riscos à saúde do trabalhador, apesar de realizarem cenários de impactos aos meios físico e biótico.

Essas omissões parecem fazer parte do processo de enfatizar os impactos positivos (geração de empregos e impostos), numa tendência a focar no otimismo tecnológico e na chantagem por novos empregos, ignorando os acidentes de trabalho ${ }^{9,13,14}$.

Quanto à proteção da saúde do trabalhador, destaca-se o uso de tecnologias para reverter danos à saúde e de alternativas tecnológicas, planos, programas e projetos de saúde, para os quais apenas o EIA REPLAN apresentou estratégias compartilhadas para potenciais impactos, mesmo que desconsiderando os sistemas municipais de saúde.

Os EIAs REPLAN e REFINE apresentaram projetos de incorporação tecnológica com inovações no design industrial, a exemplo de novas tecnologias de controle de poluição, tecnologias de gerenciamento de resíduos, tecnologias de reciclagem de resíduos, tecnologias limpas na produção de resíduos, tecnologias de geração de produtos limpões e tecnologias limpadoras.

Deve-se ressaltar que essas incorporações aumentam a competitividade das refinarias no mercado internacional, que exige produtos com baixo teor de enxofre, ou seja, a prioridade não parece ser proteger os trabalhadores.

A ausência de planos de emergência para acidentes ampliados foi verificada em todos os EIAs, e as medidas de segurança propostas são referências às normas regulamentares do Ministério do Trabalho, a programas de doação de sangue, de educação ambiental e de prevenção à exposição ocupacional, sem a garantia de recursos para a realização nem a apresentação de métodos a serem utilizados.

Deveriam apresentar programas de atenção à saúde com proposta de monitoramento, aplicação de indicadores de acompanhamento de riscos e uso de biomarcadores sensíveis à prevenção do benzenismo, identificando precocemente danos à saúde.

Constata-se um mecanismo de postergação para os riscos, além da falta de prioridade para as questões sociais, pela não inserção da saúde do trabalhador ${ }^{18,19}$.

Os resultados apontam para a necessidade de inserção nos EIAs de conteúdos importantes na produção de conhecimentos, que permitam intervenções antecipadas, tendo como paradigma a promoção da saúde e utilizando a disseminação de informações para a mobilização social e para a comunicação de risco diante de situações complexas. 


\section{REFERÊNCIAS}

1. Augusto LGS. Aviso aos navegantes. In: Augusto LGS, editor. Saúde do trabalhador no desenvolvimento humano local: ensaios em Pernambuco. Recife: Ed. Universitária UFPE; 2009. p. 17-9.

2. Nehmy RMQ, Dias EC. Os caminhos da saúde do trabalhador: para onde apontam os sinais? Revista Médica de Minas Gerais. 2010;20(2):S5-S12.

3. Brasil. Ministério da Saúde. Competência da Saúde na área de saúde ambiental: análise sobre as necessidades de inclusão de mecanismos legais complementares aos já existentes nas Leis 8.080/90 e 8.142/90. Parecer jurídico. Consultor: Paulo Affonso Leme Machado. Brasília, 2004; p. 1-70.

4. Brasil. Cartilha de licenciamento ambiental. Brasília: Tribunal de Contas da União; 2007.

5. Organização Panamericana de Saúde. Manual Básico de Avaliação do Impacto no Ambiente e na Saúde. México: OPAS; 1996.

6. Organização Mundial de Saúde. Health impact assessment as part of strategic environmental Assessment. Geneva: Regional Office for Europe, 2001.

7. Brasil. Conselho Federal de Economia [Internet]. Recursos do PAC privilegiam o setor energético [citado em 6 ago. 2010]. Disponível em: http://www.cofecon.org.br

8. Freitas CM, Porto MFS, Gomes CM. Acidentes químicos ampliados: um desafio para a saúde pública. Rev Saúde Pública. 2001;21(2):503-14.

9. Souza CAV, Freitas CM. Perfil dos acidentes de trabalho em refinaria de petróleo. Rev Saúde Pública. São Paulo. 2003;36(5):576-83.

10. Barbosa FL. Regulamentação do reuso da água em refinaria: análise do modelo americano e perspectivas para o cenário nacional [tese]. Rio de Janeiro (RJ): Universidade Federal do Rio de Janeiro; 2007.

11. Mariano JB. Impactos ambientais do refino de petróleo [tese]. Rio de Janeiro (RJ): Universidade Federal do Rio de Janeiro; 2001.

12. Sevá Filho AO. Problemas intrínsecos e graves da expansão mineral, metarlúgica, petrolífera e hidroelétrica nas Amazônias. In: Zhouri A, Laschefski K, editors. Desenvolvimento e conflitos ambientais. Belo Horizonte: Ed. UFMG; 2010. p. 114-47.
13. Augusto LGS. Estudo das alterações morfológicas (medula óssea) em portadores de neutropenia secundária à exposição ao benzeno [tese]. Campinas (SP): Universidade de Campinas; 1991.

14. Augusto LGS. Exposição ocupacional a organoclorados em indústria química de Cubatão-Estado de São Paulo: avaliação do efeito clastogêncio pelo Teste de Micronúcleos [tese]. Campinas (SP): Universidade de Campinas; 1995.

15. Ferreira LL, Iguti AM. O trabalho dos petroleiros: perigoso, complexo, contínuo e coletivo. São Paulo: Scritta; 1996.

16. Cancio JA. Inserção das questões de saúde no estudo do impacto ambiental. 2008 [Dissertação]. Brasília (DF): Universidade Católica de Brasília; 2008.

17. Porto MFS, Milanez B. Eixos de desenvolvimento econômico e geração de conflitos socioambientais no Brasil: desafios para a sustentabilidade e a justiça social. Ciên Saúde Coletiva. 2009;14(6):1983-1994.

18. Silva JM, Gurgel AM, Gurgel IDG, Augusto LGS. A inter-relação saúde, trabalho e ambiente no licenciamento da refinaria do nordeste. Tempus: Actas de Saúde Coletiva. 2009;4(4):72-83.

19. Augusto LGS. A exposição a solventes clorados e o princípio da precaução. In: Augusto LGS, editor. Saúde do Trabalhador no Desenvolvimento Humano Local: ensaios em Pernambuco. Recife: Ed. Universitária UFPE; 2009. p. 26-47.

20. Porto MFS. Uma Ecologia Política dos Riscos: princípios para integrarmos o local e o global na promoção da saúde e da justiça ambiental. Rio de Janeiro: Ed. Fiocruz; 2007.

21. Rattner H. Ambiente, Desenvolvimento e Crescimento. In: Augusto LGS, editor. Saúde do Trabalhador e Sustentabilidade do Desenvolvimento local: ensaios em Pernambuco. Recife: Ed. Universitária UFPE; 2009. p. 90-9. 